\title{
A SUPPLEMENT TO PRECISE ASYMPTOTICS IN THE LAW OF THE ITERATED LOGARITHM FOR SELF-NORMALIZED SUMS
}

\author{
KYO-SHIN HWANG
}

\begin{abstract}
Let $X, X_{1}, X_{2}, \ldots$ be i.i.d. random variables with zero means, variance one, and set $S_{n}=\sum_{i=1}^{n} X_{i}, n \geq 1$. Gut and Spătaru [3] established the precise asymptotics in the law of the iterated logarithm and Li, Nguyen and Rosalsky [7] generalized their result under minimal conditions. If $\mathbf{P}\left(\left|S_{n}\right| \geq \varepsilon \sqrt{2 n \log \log n}\right)$ is replaced by $\mathbf{E}\left\{\left|S_{n}\right| / \sqrt{n}-\right.$ $\varepsilon \sqrt{2 \log \log n}\}+$ in their results, the new one is called the moment version of precise asymptotics in the law of the iterated logarithm. We establish such a result for self-normalized sums, when $X$ belongs to the domain of attraction of the normal law.
\end{abstract}

\section{Introduction and main result}

Let $X, X_{1}, X_{2}, \ldots$ be i.i.d. random variables with $\mathbf{E} X=0$ and $S_{n}=$ $\sum_{i=1}^{n} X_{i}$. Also let $\log x=\ln (x \vee e)$ and $\log \log x=\log (\log x)$. Hsu and Robbins [5] established the well-known complete convergence, if $\mathbf{E} X^{2}<\infty$, then

$$
\sum_{n=1}^{\infty} \mathbf{P}\left(\left|S_{n}\right| \geq \varepsilon n\right)<\infty, \quad \forall \varepsilon>0
$$

Katz [6] extended this result as follows: If $\alpha>1 / 2, \alpha p \geq 1$ and $\mathbf{E}|X|^{p}<\infty$, then

$$
\sum_{n=1}^{\infty} n^{\alpha p-2} \mathbf{P}\left(\left|S_{n}\right| \geq \varepsilon n^{\alpha}\right)<\infty, \quad \forall \varepsilon>0 .
$$

Many authors considered various extensions of the results of Hsu-Robbins and Katz. Some of them study the precise asymptotics of the infinite sums as $\varepsilon \rightarrow 0$ (c.f. Heyde [4] and Spătaru [9]). But, this kind of results do not hold for $\alpha=1 / 2$. However, by replacing $n^{\alpha}$ by $\sqrt{n \log \log n}$, Gut and Spătaru [3]

Received January 23, 2007; Revised January 4, 2008.

2000 Mathematics Subject Classification. 60F15, 62E20.

Key words and phrases. precise asymptotics, law of iterated logarithm, self-normalized sums.

This work was supported by the Korea Research Foundation Grant funded by Korea Government (MOEHRD) (KRF-2006-353-C00004). 
established the following result called the precise asymptotics of the law of the iterated logarithm.

Theorem A. Suppose that $\mathbf{E} X^{2}=1$ and $\mathbf{E} X^{2}(\log \log |X|)^{1+\delta}<\infty$ for some $\delta>0$, and that $a_{n}=O\left(\sqrt{n} /(\log \log n)^{h}\right)$ for some $h>1 / 2$. Then

$$
\lim _{\varepsilon \searrow 1} \sqrt{\varepsilon^{2}-1} \sum_{n=1}^{\infty} \frac{1}{n} \mathbf{P}\left(\left|S_{n}\right| \geq \varepsilon \sqrt{2 n \log \log n}+a_{n}\right)=1 .
$$

Li, Nguyen, and Rosalsky [7] generalized this result under minimal conditions as follows:

Theorem B. Suppose that $-1 / 2<b \leq 1$, that $\mathbf{E} X^{2}=1$ and $\mathbf{E} X^{2} I\{|X| \geq$ $t\}=o\left(\frac{1}{\log \log t}\right)$ as $t \rightarrow \infty$, and that $\left\{a_{n} ; n \geq 1\right\}$ is a sequence of real numbers such that

$$
\lim _{n \rightarrow \infty}\left(\frac{\log \log n}{n}\right)^{1 / 2} a_{n}=\gamma \in[-\infty, \infty] .
$$

Then

$$
\begin{aligned}
& \lim _{\varepsilon \searrow 1}\left(\varepsilon^{2}-1\right)^{b+1 / 2} \sum_{n=1}^{\infty} \frac{(\log \log n)^{b}}{n} \mathbf{P}\left(\left|S_{n}\right| \geq \varepsilon \sqrt{2 n \log \log n}+a_{n}\right) \\
= & e^{-\sqrt{2} \gamma} 2^{b} \sqrt{2 / \pi} \Gamma(b+1 / 2),
\end{aligned}
$$

where $\Gamma(s)=\int_{0}^{\infty} x^{s-1} e^{-x} d x, s>0$ is the gamma function.

On the other hand, compared to complete convergence, Chow [2] established a moment version of (1.1) as follows: If $p \geq 1, \alpha>1 / 2, \alpha p \geq 1$ and $\mathbf{E}\left\{|X|^{p}+\right.$ $|X| \log |X|\}<\infty$, then

$$
\sum_{n=1}^{\infty} n^{\alpha p-2-\alpha} \mathbf{E}\left\{\left|S_{n}\right|-\varepsilon n^{\alpha}\right\}_{+}<\infty, \quad \forall \varepsilon>0,
$$

where $a_{+}=\max (a, 0)$. Pang et al. [8] obtained the precise rates in the law of the logarithm for the moment convergence of i.i.d. random variables.

In this paper, we prove the following theorem, which is a moment version of (1.3) for self-normalized sums.

Throughout this paper let $\left\{X, X_{n} ; n \geq 1\right\}$ be a sequence of nondegenerate i.i.d. symmetric random variables, set $S_{n}=\sum_{i=1}^{n} X_{i}, V_{n}^{2}=\sum_{i=1}^{n} X_{i}^{2}$ and $l(x)=\mathbf{E} X^{2} I\{|X| \leq x\}$. We also let $[x]$ denote the largest integer $\leq x$, $\sum_{i=1}^{0} \eta_{i}=0$, and $A$ denote a positive constant, whose values can differ in different places. $a_{n} \sim b_{n}$ means that $a_{n} / b_{n} \rightarrow 1$ as $n \rightarrow \infty$.

Theorem 1.1. Suppose that $a>-1$, that $l(x)$ is a slowly varying function at $\infty$, satisfying $l(x) \leq c_{1} \exp \left(c_{2}(\log x)^{\beta}\right)$ for some $c_{1}>0, c_{2}>0$ and $0 \leq \beta<1$, and that $\alpha_{n}(\varepsilon)$ is a nonnegative function of $\varepsilon$ such that

$$
\alpha_{n}(\varepsilon) \log \log n \rightarrow \tau<\infty \quad \text { as } n \rightarrow \infty .
$$


(1.6)

$$
\begin{aligned}
& \lim _{\varepsilon \searrow \sqrt{1+a}} \frac{1}{-\log \left(\varepsilon^{2}-1-a\right)} \sum_{n=1}^{\infty} \frac{(\log n)^{a}}{n} \mathbf{E}\left\{\left|S_{n}\right| / V_{n}-\sqrt{2 \log \log n}\left(\varepsilon+\alpha_{n}(\varepsilon)\right)\right\}_{+} \\
= & \frac{\exp (-2 \tau \sqrt{1+a})}{\sqrt{2 \pi}(1+a)} .
\end{aligned}
$$

If $b>0$, then

$$
\begin{aligned}
& \lim _{\varepsilon \searrow \sqrt{1+a}}\left(\varepsilon^{2}-a-1\right)^{b} \sum_{n=1}^{\infty} \frac{(\log n)^{a}(\log \log n)^{b}}{n} \mathbf{E}\left\{\left|S_{n}\right| / V_{n}-\sqrt{2 \log \log n}\left(\varepsilon+\alpha_{n}(\varepsilon)\right)\right\}_{+} \\
= & \frac{\Gamma(b)}{\sqrt{2 \pi}(1+a)} \exp \{-2 \tau \sqrt{1+a}\} .
\end{aligned}
$$

Remark 1.1. Note that $X$ belonging to the domain of attraction of the normal law is well known to be equivalent to $l(x)$ being a slowly varying function at $\infty$. We note also that $l(x) \leq c_{1} \exp \left(c_{2}(\log x)^{\beta}\right)$ is a weak enough assumption, which is satisfied by a large class of slowly varying functions such as $(\log \log x)^{p}$ and $(\log x)^{p}$ for some $0<p<\infty$.

\section{The proof of Theorem 1.1}

For convenience, we need some notation. Put $c=\inf \{x \geq 1: l(x)>0\}$ and

$$
\eta_{n}=\inf \left\{s: s \geq c+1, \frac{l(s)}{s^{2}} \leq \frac{(\log \log n)^{2}}{n}\right\}
$$

Furthermore, for each $n$ and $1 \leq i \leq n$, we let

$$
\begin{array}{lc}
\bar{X}_{n i}=X_{i} I\left\{\left|X_{i}\right| \leq \eta_{n}\right\}, & \bar{S}_{n}=\sum_{i=1}^{n} \bar{X}_{n i}, \quad \bar{V}_{n}^{2}=\sum_{i=1}^{n} \bar{X}_{n i}^{2}, \\
S_{n}^{i}=S_{n}-X_{i}, & V_{n}^{i}=\left(V_{n}^{2}-X_{i}^{2}\right)^{1 / 2}, \\
\bar{S}_{n}^{i}=\bar{S}_{n}-\bar{X}_{n i}, & \bar{V}_{n}^{i}=\left(\bar{V}_{n}^{2}-\bar{X}_{n i}^{2}\right)^{1 / 2} .
\end{array}
$$

Note that $n l\left(\eta_{n}\right) \sim \eta_{n}^{2}(\log \log n)^{2}$. Thus we have

$$
l\left(\eta_{n}\right) \leq c_{1} \exp \left(c_{2}\left(\log \eta_{n}\right)^{\beta}\right) \leq c_{1} \exp \left(c_{2}(\log n)^{\beta}\right)
$$

for $n$ large enough, and we also have that $l\left(\eta_{n}\right)$ and $c_{1} \exp \left(c_{2}(\log n)^{\beta}\right) / l\left(\eta_{n}\right)$ are slowly varying functions at $\infty$ (see [1, Chapter 1$])$. Using these facts, it follows easily that

$$
c_{1} \exp \left(c_{2}(\log j)^{\beta}\right) / l\left(\eta_{j}\right) \geq \frac{1}{2} c_{1} \exp \left(c_{2}(\log k)^{\beta}\right) / l\left(\eta_{k}\right)
$$


for all $j \geq k$ and $k$ large enough, and hence

$$
\begin{aligned}
& \frac{A}{l\left(\eta_{k}\right)(\log k)^{\beta}(\log \log k)^{2}} \\
\leq & \frac{\exp \left(c_{2}(\log k)^{\beta}\right)}{2 l\left(\eta_{k}\right)} \sum_{j=k}^{\infty} \frac{1}{j \exp \left(c_{2}(\log j)^{\beta}\right)(\log j)(\log \log j)^{2}} \\
\leq & \sum_{j=k}^{\infty} \frac{1}{j l\left(\eta_{j}\right)(\log j)(\log \log j)^{2}} .
\end{aligned}
$$

We first will prove Theorem 1.1 in the case that $X, X_{1}, X_{2}, \ldots$ are normal random variables. Let $N$ be a standard normal variable, we have the following proposition.

Proposition 2.1. Let $a>-1$ and $\alpha_{n}(\varepsilon)$ be a nonnegative function of $\varepsilon$ satisfying (1.5). Then

$$
\begin{aligned}
& \lim _{\varepsilon \searrow \sqrt{1+a}} \frac{1}{-\log \left(\varepsilon^{2}-1-a\right)} \sum_{n=1}^{\infty} \frac{(\log n)^{a}}{n} \mathbf{E}\left\{|N|-\left(\varepsilon+\alpha_{n}(\varepsilon)\right) \sqrt{2 \log \log n}\right\}_{+} \\
= & \frac{\exp \{-2 \tau \sqrt{1+a}\}}{\sqrt{2 \pi}(1+a)} .
\end{aligned}
$$

If $b>0$, then

$$
\begin{aligned}
& \lim _{\varepsilon \searrow \sqrt{1+a}}\left(\varepsilon^{2}-1-a\right)^{b} \sum_{n=1}^{\infty} \frac{(\log n)^{a}(\log \log n)^{b}}{n} \mathbf{E}\left\{|N|-\left(\varepsilon+\alpha_{n}(\varepsilon)\right) \sqrt{2 \log \log n}\right\}_{+} \\
= & \frac{\Gamma(b)}{\sqrt{2 \pi}(1+a)} \exp \{-2 \tau \sqrt{1+a}\} .
\end{aligned}
$$

Proof. Let $\psi_{n}(\varepsilon)=\left(\varepsilon+\alpha_{n}(\varepsilon)\right) \sqrt{2 \log \log n}$. Note that the limit in (2.2) and (2.3) does not depend on any finite terms of the infinite series, and we have

$$
\mathbf{P}(|N| \geq x) \sim \frac{2}{\sqrt{2 \pi} x} e^{-x^{2} / 2} \quad \text { as } \quad x \rightarrow \infty .
$$

Consider (2.2), for any $x>0$, by (1.5)

$$
\begin{aligned}
& \mathbf{P}\left(|N| \geq x+\psi_{n}(\varepsilon)\right) \\
\sim & \frac{2}{\sqrt{2 \pi}\left(x+\psi_{n}(\varepsilon)\right)} \exp \left\{-\frac{1}{2}\left(x+\psi_{n}(\varepsilon)\right)^{2}\right\} \\
\sim & \frac{2}{\sqrt{2 \pi}} \frac{1}{x+\varepsilon \sqrt{2 \log \log n}} \exp \left\{-\frac{1}{2}(x+\varepsilon \sqrt{2 \log \log n})^{2}\right\} \\
& \times \exp \left\{-2 \varepsilon \alpha_{n}(\varepsilon) \log \log n-x \alpha_{n}(\varepsilon) \sqrt{2 \log \log n}\right\} \quad \text { as } n \rightarrow \infty,
\end{aligned}
$$


THE LAW OF THE ITERATED LOGARITHM FOR SELF-NORMALIZED SUMS 1605

uniformly in $\varepsilon \in(\sqrt{1+a}, \sqrt{1+a}+\delta)$ for some $\delta>0$. So, for any $x>0$ and $0<\theta<1$, there exist $\delta>0$ and $n_{0}$ such that for all $n \geq n_{0}$ and $\varepsilon \in(\sqrt{1+a}, \sqrt{1+a}+\delta)$,

(2.4)

$$
\begin{aligned}
& \frac{2}{\sqrt{2 \pi}} \frac{1}{x+\varepsilon \sqrt{2 \log \log n}} \exp \left\{-\frac{1}{2}(x+\varepsilon \sqrt{2 \log \log n})^{2}\right\} \exp \{-2 \tau \sqrt{1+a}-\theta\} \\
\leq & \mathbf{P}\left(|N| \geq x+\psi_{n}(\varepsilon)\right) \\
\leq & \frac{2}{\sqrt{2 \pi}} \frac{1}{x+\varepsilon \sqrt{2 \log \log n}} \exp \left\{-\frac{1}{2}(x+\varepsilon \sqrt{2 \log \log n})^{2}\right\} \exp \{-2 \tau \sqrt{1+a}+\theta\} .
\end{aligned}
$$

Write

$$
\begin{aligned}
& \lim _{\varepsilon \backslash \sqrt{1+a}} \frac{1}{-\log \left(\varepsilon^{2}-1-a\right)} \sum_{n=1}^{\infty} \frac{(\log n)^{a}}{n} \mathbf{E}\left\{|N|-\psi_{n}(\varepsilon)\right\}_{+} \\
= & \lim _{\varepsilon \searrow \sqrt{1+a}} \frac{1}{-\log \left(\varepsilon^{2}-1-a\right)} \int_{e^{e}}^{\infty} \frac{(\log y)^{a}}{y} \int_{0}^{\infty} \mathbf{P}\left(|N| \geq x+\psi_{y}(\varepsilon)\right) d x d y
\end{aligned}
$$

and

$$
\begin{aligned}
& \lim _{\varepsilon \searrow \sqrt{1+a}} \frac{1}{-\log \left(\varepsilon^{2}-1-a\right)} \int_{e^{e}}^{\infty} \frac{(\log y)^{a}}{y} \int_{0}^{\infty} \frac{\exp \left\{-\frac{1}{2}(x+\varepsilon \sqrt{2 \log \log y})^{2}\right\}}{x+\varepsilon \sqrt{2 \log \log y}} d x d y \\
= & \lim _{\varepsilon \searrow \sqrt{1+a}} \frac{1}{-\log \left(\varepsilon^{2}-1-a\right)} \int_{e^{e}}^{\infty} \frac{(\log y)^{a}}{y} \int_{\varepsilon \sqrt{2 \log \log y}}^{\infty} \frac{1}{x} \exp \left\{-\frac{1}{2} x^{2}\right\} d x d y \\
= & \lim _{\varepsilon \searrow \sqrt{1+a}} \frac{1}{-\log \left(\varepsilon^{2}-1-a\right)} \int_{\sqrt{2} \varepsilon}^{\infty} \frac{t}{\varepsilon^{2}} \exp \left\{\frac{1+a}{2 \varepsilon^{2}} t^{2}\right\} \int_{t}^{\infty} \frac{1}{x} \exp \left\{-\frac{1}{2} x^{2}\right\} d x d t \\
= & \frac{1}{1+a} \lim _{\varepsilon \backslash \sqrt{1+a}} \frac{1}{-\log \left(\varepsilon^{2}-1-a\right)} \int_{\sqrt{2} \varepsilon}^{\infty} \frac{1}{x} \exp \left\{-\frac{1}{2} x^{2}\right\}\left[\exp \left\{\frac{1+a}{2 \varepsilon^{2}} x^{2}\right\}-e^{1+a}\right] d x \\
= & \frac{1}{2(1+a)} \varepsilon \searrow \sqrt{1+a} \frac{1}{-\log \left(\varepsilon^{2}-1-a\right)} \int_{\varepsilon^{2}-1-a}^{\infty} \frac{1}{s} e^{-s} d s \\
= & \frac{1}{2(1+a)} \varepsilon \backslash \sqrt{1+a} \frac{1}{-\log \left(\varepsilon^{2}-1-a\right)}\left[\int_{\varepsilon^{2}-1-a}^{1} \frac{1}{s} e^{-s} d s+\int_{1}^{\infty} \frac{1}{s} e^{-s} d s\right] \\
= & \frac{1}{2(1+a)} .
\end{aligned}
$$

Hence, from (2.4) (2.6), (2.2) is obtained by letting $\theta \rightarrow 0$.

We now proceed to show (2.3). This follows by the same method as in (2.5) and (2.6)

$$
\begin{aligned}
& \lim _{\varepsilon \backslash \sqrt{1+a}}\left(\varepsilon^{2}-1-a\right)^{b} \sum_{n=1}^{\infty} \frac{(\log n)^{a}(\log \log n)^{b}}{n} \mathbf{E}\left\{|N|-\psi_{n}(\varepsilon)\right\}_{+} \\
= & \lim _{\varepsilon \searrow \sqrt{1+a}}\left(\varepsilon^{2}-1-a\right)^{b} \int_{e^{e}}^{\infty} \frac{(\log y)^{a}(\log \log y)^{b}}{y} \int_{0}^{\infty} \mathbf{P}\left(|N| \geq x+\psi_{y}(\varepsilon)\right) d x d y
\end{aligned}
$$




$$
\begin{aligned}
& \lim _{\varepsilon \backslash \sqrt{1+a}}\left(\varepsilon^{2}-1-a\right)^{b} \int_{e^{e}}^{\infty} \frac{(\log y)^{a}(\log \log y)^{b}}{y} \int_{0}^{\infty} \frac{\exp \left\{-\frac{1}{2}(x+\varepsilon \sqrt{2 \log \log y})^{2}\right\}}{x+\varepsilon \sqrt{2 \log \log y}} d x d y \\
= & \lim _{\varepsilon \backslash \sqrt{1+a}}\left(\varepsilon^{2}-1-a\right)^{b} \int_{\sqrt{2} \varepsilon}^{\infty} \frac{1}{x} \exp \left\{-\frac{1}{2} x^{2}\right\}\left[\int_{\sqrt{2} \varepsilon}^{x} \frac{t}{\varepsilon^{2}}\left(\frac{t^{2}}{2 \varepsilon^{2}}\right)^{b} \exp \left\{\frac{1+a}{2 \varepsilon^{2}} t^{2}\right\} d t\right] d x .
\end{aligned}
$$

If $0<b<1$, then using integration by parts

$$
\begin{aligned}
& \lim _{\varepsilon \searrow \sqrt{1+a}}\left(\varepsilon^{2}-1-a\right)^{b} \int_{\sqrt{2} \varepsilon}^{\infty} \frac{1}{x} \exp \left\{-\frac{1}{2} x^{2}\right\}\left[\int_{\sqrt{2} \varepsilon}^{x} \frac{t}{\varepsilon^{2}}\left(\frac{t^{2}}{2 \varepsilon^{2}}\right)^{b} \exp \left\{\frac{1+a}{2 \varepsilon^{2}} t^{2}\right\} d t\right] d x \\
= & \frac{1}{(1+a)^{1+b}} \lim _{\varepsilon \searrow \sqrt{1+a}}\left(\varepsilon^{2}-1-a\right)^{b} \int_{\sqrt{2} \varepsilon}^{\infty} \frac{1}{x} \exp \left\{-\frac{1}{2} x^{2}\right\} \int_{1+a}^{\frac{1+a}{2 \varepsilon^{2}} x^{2}} t^{b} e^{t} d t d x \\
= & \frac{1}{(1+a)^{1+b}} \varepsilon \lim _{\varepsilon \backslash \sqrt{1+a}}\left(\varepsilon^{2}-1-a\right)^{b} \int_{\sqrt{2} \varepsilon}^{\infty} \frac{1}{x} \exp \left\{-\frac{1}{2} x^{2}\right\}\left(\frac{1+a}{2 \varepsilon^{2}} x^{2}\right)^{b} \exp \left\{\frac{1+a}{2 \varepsilon^{2}} x^{2}\right\} d x \\
& -\frac{b}{(1+a)^{1+b}} \lim _{\varepsilon \searrow \sqrt{1+a}}\left(\varepsilon^{2}-1-a\right)^{b} \int_{\sqrt{2} \varepsilon}^{\infty} \frac{1}{x} \exp \left\{-\frac{1}{2} x^{2}\right\} \int_{1+a}^{\frac{1+a}{2 \varepsilon^{2}} x^{2}} t^{b-1} e^{t} d t d x \\
= & : I_{1}-I_{2} .
\end{aligned}
$$

We have

(2.10)

$$
\begin{aligned}
I_{1} & =\frac{1}{1+a} \lim _{\varepsilon \searrow \sqrt{1+a}}\left(\varepsilon^{2}-1-a\right)^{b} \int_{\sqrt{2} \varepsilon}^{\infty}\left(2 \varepsilon^{2}\right)^{-b} x^{2 b-1} \exp \left\{-\left(\frac{1}{2}-\frac{1+a}{2 \varepsilon^{2}}\right) x^{2}\right\} d x \\
& =\frac{1}{2(1+a)} \lim _{\varepsilon \searrow \sqrt{1+a}} \int_{\varepsilon^{2}-1-a}^{\infty} s^{b-1} e^{-s} d s \\
& =\frac{1}{2(1+a)} \Gamma(b)
\end{aligned}
$$

and by (2.6)

$$
\begin{aligned}
& 0 \leq I_{2} \leq \frac{b(1+a)^{b-1}}{(1+a)^{1+b}} \lim _{\varepsilon \searrow \sqrt{1+a}}\left(\varepsilon^{2}-1-a\right)^{b} \int_{\sqrt{2} \varepsilon}^{\infty} \frac{1}{x} \exp \left\{-\left(\frac{1}{2}-\frac{1+a}{2 \varepsilon^{2}}\right) x^{2}\right\} d x \\
& =\frac{b}{2(1+a)^{2}} \lim _{\varepsilon \searrow \sqrt{1+a}}\left(\varepsilon^{2}-1-a\right)^{b} \int_{\varepsilon^{2}-1-a}^{\infty} s^{-1} e^{-s} d s \\
& =\frac{b}{2(1+a)^{2}} \lim _{\varepsilon \searrow \sqrt{1+a}}\left[\left(\varepsilon^{2}-1-a\right)^{b} \cdot\left(-\log \left(\varepsilon^{2}-1-a\right)\right)\right] \frac{\int_{\varepsilon^{2}-1-a}^{\infty} s^{-1} e^{-s} d s}{-\log \left(\varepsilon^{2}-1-a\right)} \\
& =0 \text {. }
\end{aligned}
$$

Thus, letting $\theta \rightarrow 0$, (2.3) follows from (2.4) and $(2.7) \sim(2.11)$. 
THE LAW OF THE ITERATED LOGARITHM FOR SELF-NORMALIZED SUMS 1607

If $b \geq 1$, then we write via integration by parts again

$$
\begin{aligned}
& \int_{\sqrt{2} \varepsilon}^{x} \frac{t}{\varepsilon^{2}}\left(\frac{t^{2}}{2 \varepsilon^{2}}\right)^{b} \exp \left\{\frac{1+a}{2 \varepsilon^{2}} t^{2}\right\} d t=\frac{1}{(1+a)^{b+1}} \int_{1+a}^{\frac{1+a}{2 \varepsilon^{2}} x^{2}} t^{b} e^{t} d t \\
= & \frac{1}{(1+a)^{b+1}}\left[\left(\frac{1+a}{2 \varepsilon^{2}} x^{2}\right)^{b} \exp \left\{\frac{1+a}{2 \varepsilon^{2}} x^{2}\right\}-(1+a)^{b} e^{1+a}\right] \\
& +\frac{1}{(1+a)^{b+1}} \sum_{i=1}^{[b]-1}(-1)^{i}\left(\prod_{j=b-i+1}^{b} j\right)\left[\left(\frac{1+a}{2 \varepsilon^{2}} x^{2}\right)^{b-i} \exp \left\{\frac{1+a}{2 \varepsilon^{2}} x^{2}\right\}-(1+a)^{b-i} e^{1+a}\right] \\
& +\frac{(-1)^{[b]} \prod_{j=0}^{[b]-1}(b-j)}{(1+a)^{b+1}} \int_{1+a}^{\frac{1+a}{2 \varepsilon^{2}} x^{2}} t^{b-[b]} e^{t} d t \\
= & J_{x 1}+J_{x 2}+J_{x 3} .
\end{aligned}
$$

We have by (2.10),

$$
\lim _{\varepsilon \searrow \sqrt{1+a}}\left(\varepsilon^{2}-1-a\right)^{b} \int_{\sqrt{2} \varepsilon}^{\infty} \frac{1}{x} \exp \left\{-\frac{1}{2} x^{2}\right\} J_{x 1} d x=\frac{1}{2(1+a)} \Gamma(b),
$$

$$
\begin{aligned}
& \lim _{\varepsilon \searrow \sqrt{1+a}}\left(\varepsilon^{2}-1-a\right)^{b} \int_{\sqrt{2} \varepsilon}^{\infty} \frac{1}{x} \exp \left\{-\frac{1}{2} x^{2}\right\} J_{x 2} d x \\
&= \sum_{i=1}^{[b]-1} \frac{(-1)^{i}\left(\prod_{j=b-i+1}^{b} j\right)}{(1+a)^{1+i}} \lim _{\varepsilon \searrow \sqrt{1+a}}\left(\varepsilon^{2}-1-a\right)^{b} \\
& \times \int_{\sqrt{2} \varepsilon}^{\infty}\left(\frac{1}{2 \varepsilon^{2}}\right)^{b-i} x^{2 b-2 i-1} \exp \left\{-\left(\frac{1}{2}-\frac{1+a}{2 \varepsilon^{2}}\right) x^{2}\right\} d x \\
&=\sum_{i=1}^{[b]-1} \frac{(-1)^{i}\left(\prod_{j=b-i+1}^{b} j\right)}{2(1+a)^{1+i}} \lim _{\varepsilon \searrow \sqrt{1+a}}\left(\varepsilon^{2}-1-a\right)^{i} \int_{\varepsilon^{2}-1-a}^{\infty} s^{b-i-1} e^{-s} d s \\
&=0
\end{aligned}
$$

and

$$
\begin{aligned}
& \lim _{\varepsilon \searrow \sqrt{1+a}}\left(\varepsilon^{2}-1-a\right)^{b}\left|\int_{\sqrt{2} \varepsilon}^{\infty} \frac{1}{x} \exp \left\{-\frac{1}{2} x^{2}\right\} J_{x 3} d x\right| \\
\leq & \frac{\prod_{j=0}^{[b]-1}(b-j)}{(1+a)^{b+1}} \lim _{\varepsilon \searrow \sqrt{1+a}}\left(\varepsilon^{2}-1-a\right)^{b} \int_{\sqrt{2} \varepsilon}^{\infty}\left(\frac{1+a}{2 \varepsilon^{2}}\right)^{b-[b]} x^{2 b-2[b]-1} \exp \left\{-\left(\frac{1}{2}-\frac{1+a}{2 \varepsilon^{2}}\right) x^{2}\right\} d x \\
= & \frac{\prod_{j=0}^{[b]-1}(b-j)}{2(1+a)^{[b]+1}} \lim _{\varepsilon \searrow \sqrt{1+a}}\left(\varepsilon^{2}-1-a\right)^{[b]} \int_{\varepsilon^{2}-1-a}^{\infty} s^{b-[b]-1} e^{-s} d s \\
= & 0 .
\end{aligned}
$$

Therefore, letting $\theta \rightarrow 0,(2.3)$ follows from (2.4), (2.7), (2.8) and (2.12) (2.15), which completes the proof. 
The following is the proof of Theorem 1.1 in general case via the non-uniform Berry-Esseen bound for self-normalized sums.

Proof of Theorem 1.1. We will only verify (1.7) since the proof of (1.6) is similar. Let $\psi_{n}(\varepsilon)=\sqrt{2 \log \log n}\left(\varepsilon+\alpha_{n}(\varepsilon)\right)$. By Proposition 2.1 (2.3), it suffices to prove that

$$
\begin{array}{r}
\lim _{\varepsilon \backslash \sqrt{1+a}}\left(\varepsilon^{2}-a-1\right)^{b} \sum_{n=1}^{\infty} \frac{(\log n)^{a}(\log \log n)^{b}}{n} \mid \mathbf{E}\left\{\left|S_{n}\right| / V_{n}-\psi_{n}(\varepsilon)\right\}_{+} \\
-\mathbf{E}\left\{|N|-\psi_{n}(\varepsilon)\right\}_{+} \mid=0 .
\end{array}
$$

Write

and

$$
\begin{aligned}
& \left|\mathbf{E}\left\{\left|S_{n}\right| / V_{n}-\psi_{n}(\varepsilon)\right\}_{+}-\mathbf{E}\left\{|N|-\psi_{n}(\varepsilon)\right\}_{+}\right| \\
\leq & \int_{0}^{\infty}\left|\mathbf{P}\left(\frac{\left|S_{n}\right|}{V_{n}} \geq x+\psi_{n}(\varepsilon)\right)-\mathbf{P}\left(|N| \geq x+\psi_{n}(\varepsilon)\right)\right| d x
\end{aligned}
$$

$$
\begin{aligned}
& \left|\mathbf{P}\left(\frac{\left|S_{n}\right|}{V_{n}} \geq x+\psi_{n}(\varepsilon)\right)-\mathbf{P}\left(|N| \geq x+\psi_{n}(\varepsilon)\right)\right| \\
\leq & \left|\mathbf{P}\left(\frac{\left|S_{n}\right|}{V_{n}} \geq x+\psi_{n}(\varepsilon)\right)-\mathbf{P}\left(\frac{\left|\bar{S}_{n}\right|}{\bar{V}_{n}} \geq x+\psi_{n}(\varepsilon)\right)\right| \\
& +\left|\mathbf{P}\left(\frac{\left|\bar{S}_{n}\right|}{\bar{V}_{n}} \geq x+\psi_{n}(\varepsilon)\right)-\mathbf{P}\left(|N| \geq x+\psi_{n}(\varepsilon)\right)\right| \\
\leq & \left|\mathbf{P}\left(\frac{S_{n}}{V_{n}} \geq x+\psi_{n}(\varepsilon)\right)-\mathbf{P}\left(\frac{\bar{S}_{n}}{\bar{V}_{n}} \geq x+\psi_{n}(\varepsilon)\right)\right| \\
& +\left|\mathbf{P}\left(-\frac{S_{n}}{V_{n}} \geq x+\psi_{n}(\varepsilon)\right)-\mathbf{P}\left(-\frac{\bar{S}_{n}}{\bar{V}_{n}} \geq x+\psi_{n}(\varepsilon)\right)\right| \\
& +\left|\mathbf{P}\left(\frac{\left|\bar{S}_{n}\right|}{\bar{V}_{n}} \geq x+\psi_{n}(\varepsilon)\right)-\mathbf{P}\left(|N| \geq x+\psi_{n}(\varepsilon)\right)\right| \\
=: & K_{x 1}+K_{x 2}+K_{x 3} .
\end{aligned}
$$

To prove (2.16), it suffices to show that

(2.17)

$$
\lim _{\varepsilon \searrow \sqrt{1+a}}\left(\varepsilon^{2}-a-1\right)^{b} \sum_{n=1}^{\infty} \frac{(\log n)^{a}(\log \log n)^{b}}{n} \int_{0}^{\infty} K_{x i} d x=0, \quad i=1,2,3 .
$$

Note that for any $s, t \in R, c \geq 0$ and $x \geq 1$,

$$
\begin{aligned}
x \sqrt{c+t^{2}} & =\sqrt{\left(x^{2}-1\right) c+t^{2}+c+\left(x^{2}-1\right) t^{2}} \\
& \geq \sqrt{\left(x^{2}-1\right) c+t^{2}+2 t \sqrt{\left(x^{2}-1\right) c}} \\
& =t+\sqrt{\left(x^{2}-1\right) c} .
\end{aligned}
$$

Thus we have

$$
\left\{s+t \geq x \sqrt{c+t^{2}}\right\} \subset\left\{s \geq\left(x^{2}-1\right)^{1 / 2} \sqrt{c}\right\}
$$


THE LAW OF THE ITERATED LOGARITHM FOR SELF-NORMALIZED SUMS 1609

Hence, for any $x>0$

$$
\begin{aligned}
K_{x 1} \leq & \mathbf{P}\left(S_{n} \geq V_{n}\left(x+\psi_{n}(\varepsilon)\right), \max _{1 \leq i \leq n}\left|X_{i}\right|>\eta_{n}\right) \\
& +\mathbf{P}\left(\bar{S}_{n} \geq \bar{V}_{n}\left(x+\psi_{n}(\varepsilon)\right), \max _{1 \leq i \leq n}\left|X_{i}\right|>\eta_{n}\right) \\
\leq & \sum_{i=1}^{n} \mathbf{P}\left(S_{n}^{(i)} \geq\left(\left(x+\psi_{n}(\varepsilon)\right)^{2}-1\right)^{1 / 2} V_{n}^{(i)},\left|X_{i}\right|>\eta_{n}\right) \\
& +\sum_{i=1}^{n} \mathbf{P}\left(\bar{S}_{n}^{(i)} \geq\left(\left(x+\psi_{n}(\varepsilon)\right)^{2}-1\right)^{1 / 2} \bar{V}_{n}^{(i)},\left|X_{i}\right|>\eta_{n}\right) \\
\leq & \sum_{i=1}^{n} \mathbf{P}\left(S_{n}^{(i)} \geq\left(\left(x+\psi_{n}(\varepsilon)\right)^{2}-1\right)^{1 / 2} V_{n}^{(i)}\right) \mathbf{P}\left(\left|X_{i}\right|>\eta_{n}\right) \\
& +\sum_{i=1}^{n} \mathbf{P}\left(\bar{S}_{n}^{(i)} \geq\left(\left(x+\psi_{n}(\varepsilon)\right)^{2}-1\right)^{1 / 2} \bar{V}_{n}^{(i)}\right) \mathbf{P}\left(\left|X_{i}\right|>\eta_{n}\right) .
\end{aligned}
$$

Notice that $\eta_{n}^{2} \sim n l\left(\eta_{n}\right) /(\log \log n)^{2}$ and $l\left(\eta_{n}\right)$ is a slowly varying function at $\infty$. From (1.5), (2.1), (2.18), Lemma 4.3 in [10] we have for some $0 \leq \beta<1$

$$
\begin{aligned}
& \lim _{\varepsilon \searrow \sqrt{1+a}}\left(\varepsilon^{2}-a-1\right)^{b} \sum_{n=1}^{\infty} \frac{(\log n)^{a}(\log \log n)^{b}}{n} \int_{0}^{\infty} K_{x 1} d x \\
= & \lim _{\varepsilon \searrow \sqrt{1+a}}\left(\varepsilon^{2}-a-1\right)^{b} \sum_{n=1}^{\infty} \frac{(\log n)^{a}(\log \log n)^{b}}{n}\left[\int_{0}^{\psi_{n}(\varepsilon)}+\int_{\psi_{n}(\varepsilon)}^{\infty}\right] K_{x 1} d x \\
\leq & A \lim _{\varepsilon \searrow \sqrt{1+a}}\left(\varepsilon^{2}-a-1\right)^{b} \sum_{n=1}^{\infty}(\log n)^{a}(\log \log n)^{b-1 / 2}(\log n)^{-\left(\varepsilon+\alpha_{n}(\varepsilon)\right)^{2}} \mathbf{P}\left(|X|>\eta_{n}\right) \\
& +A \lim _{\varepsilon \searrow \sqrt{1+a}}\left(\varepsilon^{2}-a-1\right)^{b} \sum_{n=1}^{\infty}(\log n)^{a}(\log \log n)^{b} \int_{\psi_{n}(\varepsilon)}^{\infty} \exp \left\{-\frac{\left(x+\psi_{n}(\varepsilon)\right)^{2}}{2}\right\} d x \mathbf{P}\left(|X|>\eta_{n}\right) \\
\leq & A \lim _{\varepsilon \searrow \sqrt{1+a}}\left(\varepsilon^{2}-a-1\right)^{b} \sum_{n=1}^{\infty}(\log n)^{-1}(\log \log n)^{b-1 / 2} \mathbf{P}\left(|X|>\eta_{n}\right) \\
\leq & A \lim _{\varepsilon \searrow \sqrt{1+a}}\left(\varepsilon^{2}-a-1\right)^{b} \sum_{k=1}^{\infty} \eta_{k}^{-2} \mathbf{E} X^{2} I\left\{\eta_{k}<|X| \leq \eta_{k+1}\right\} \sum_{n=1}^{k}(\log n)^{-1}(\log \log n)^{b-1 / 2} \\
\leq & A \lim _{\varepsilon \searrow \sqrt{1+a}}\left(\varepsilon^{2}-a-1\right)^{b} \sum_{k=1}^{\infty} \frac{1}{l\left(\eta_{k}\right)(\log k)^{\beta}(\log \log k)^{2}} \mathbf{E} X^{2} I\left\{\eta_{k}<|X| \leq \eta_{k+1}\right\} \\
\leq & A \lim _{\varepsilon \searrow \sqrt{1+a}}\left(\varepsilon^{2}-a-1\right)^{b} \sum_{j=1}^{\infty} \frac{\mathbf{E} X^{2} I\left\{|X| \leq \eta_{j+1}\right\}}{j l\left(\eta_{j}\right)(\log j)(\log \log j)^{2}}=0 .
\end{aligned}
$$

Similarly,

$$
\lim _{\varepsilon \searrow \sqrt{1+a}}\left(\varepsilon^{2}-a-1\right)^{b+1 / 2} \sum_{n=1}^{\infty} \frac{(\log n)^{a}(\log \log n)^{b}}{n} \int_{0}^{\infty} K_{x 2} d x=0 .
$$


From Corollary 2.1 in [10] and (2.1), for some $0 \leq \beta<1$

$$
\begin{aligned}
& \lim _{\varepsilon \searrow \sqrt{1+a}}\left(\varepsilon^{2}-a-1\right)^{b} \sum_{n=1}^{\infty} \frac{(\log n)^{a}(\log \log n)^{b}}{n} \int_{0}^{\infty} K_{x 3} d x \\
= & \lim _{\varepsilon \searrow \sqrt{1+a}}\left(\varepsilon^{2}-a-1\right)^{b} \sum_{n=1}^{\infty} \frac{(\log n)^{a}(\log \log n)^{b}}{n}\left[\int_{0}^{\psi_{n}(\varepsilon)}+\int_{\psi_{n}(\varepsilon)}^{\infty}\right] K_{x 3} d x \\
\leq & A \lim _{\varepsilon \searrow \sqrt{1+a}}\left(\varepsilon^{2}-a-1\right)^{b} \sum_{n=1}^{\infty}(\log n)^{a-\left(\varepsilon+\alpha_{n}(\varepsilon)\right)^{2}}(\log \log n)^{b+2} \frac{\mathbf{E}|X|^{3} I\left\{|X| \leq \eta_{n}\right\}}{n^{3 / 2}\left(l\left(\eta_{n}\right)\right)^{3 / 2}} \\
\leq & A \lim _{\varepsilon \searrow \sqrt{1+a}}\left(\varepsilon^{2}-a-1\right)^{b} \sum_{k=1}^{\infty} \eta_{k} \mathbf{E} X^{2} I\left\{\eta_{k-1}<|X| \leq \eta_{k}\right\} \sum_{n=k}^{\infty} \frac{(\log \log n)^{b+2}}{n^{3 / 2}(\log n)\left(l\left(\eta_{n}\right)\right)^{3 / 2}} \\
\leq & A \lim _{\varepsilon \searrow \sqrt{1+a}}\left(\varepsilon^{2}-a-1\right)^{b} \sum_{k=1}^{\infty} \frac{1}{l\left(\eta_{k}\right)(\log k)^{\beta}(\log \log k)^{2}} \mathbf{E} X^{2} I\left\{\eta_{k-1}<|X| \leq \eta_{k}\right\} \\
\leq & A \lim _{\varepsilon \searrow \sqrt{1+a}}\left(\varepsilon^{2}-a-1\right)^{b} \sum_{j=1}^{\infty} \frac{\mathbf{E} X^{2} I\left\{|X| \leq \eta_{j}\right\}}{j l\left(\eta_{j}\right)(\log j)(\log \log j)^{2}}=0 .
\end{aligned}
$$

The proof is complete.

Acknowledgments. The author would like to express his gratitude to the referee for his/her insightful comments and invaluable suggestions, and the author gratefully acknowledges the many helpful suggestions of Dr. Wang during the preparation of the paper.

\section{References}

[1] N. H. Bingham, C. M. Goldie, and J. L. Teugels, Regular Variation, Encyclopedia of Mathematics and its Applications, Vol. 27. Cambridge University Press, Cambridge, 1987.

[2] Y. S. Chow, On the rate of moment convergence of sample sums and extremes, Bull. Inst. Math. Acad. Sin. (N.S.) 16 (1988), no. 3, 177-201.

[3] A. Gut and A. Spătaru, Precise asymptotics in the law of the iterated logarithm, Ann. Probab. 28 (2000), no. 4, 1870-1883.

[4] C. C. Heyde, A supplement to the strong law of large numbers, J. Appl. Probab. 12 (1975), no. 1, 173-175.

[5] P. L. Hsu and H. Robbins, Complete convergence and the law of large numbers, Proc. Natl. Acad. Sci. USA 33 (1947), no. 2, 25-31.

[6] M. L. Katz, The probability in the tail of a distribution, Ann. Math. Statist. 34 (1963), no. $1,312-318$.

[7] D. Li, B.-E. Nguyen, and A. Rosalsky, A supplement to precise asymptotics in the law of the iterated logarithm, J. Math. Anal. Appl. 302 (2005), no. 1, 84-96.

[8] T.-X. Pang, Z.-Y. Lin, Y. Jiang, and K.-S. Hwang, Precise rates in the law of the logarithm for the moment convergence of i.i.d. random variables, J. Koran Math. Soc. 45 (2008), no. 4, 993-1005.

[9] A. Spătaru, Precise asymptotics in Spitzer's law of large numbers, J. Theoret. Probab. 12 (1999), no. 3, 811-819. 
THE LAW OF THE ITERATED LOGARITHM FOR SELF-NORMALIZED SUMS 1611

[10] Q. Wang and B. Y. Jing, An exponential nonuniform Berry-Esseen bound for selfnormalized sums, Ann. Probab. 27 (1999), no. 4, 2068-2088.

The Research Institute of Natural Science

Gyeongsang National University

JINJU 660-701, KOREA

E-mail address: hwang0412@naver.com 